\title{
Tunable Laser Stark-Difference Spectra of $\mathrm{CD}_{3} \mathrm{OH}$
}

\author{
W. H. WEBER \\ Physics Department, Engineering and Research Staff. Ford Motor Company, \\ Dearborn, Michigan 48121 \\ D. H. LesLiE \\ Naval Research Laboratory, 4555 Overlook Ave., S. W., Washington, D. C. 20375
}

AND

C. W. Peters

Physics Department, University of Michigan, Ann Arbor, Michigan 48109

\begin{abstract}
We present Doppler resolution limited spectra of the $P(J)$ and $R(J)$ multiplets for $J \leqq 10$ of the $10-\mu \mathrm{m} \mathrm{C}-\mathrm{O}$ stretch band of ${ }^{12} \mathrm{CD}_{3}{ }^{16} \mathrm{OH}$ using a tunable diode laser. Relative frequencies within the multiplets accurate to $\pm 0.0002-0.0005 \mathrm{~cm}^{-1}$ are obtained, but no absolute frequencies are given. We are able to assign most of the hindered rotation and $K$ substructure in these multiplets. The assignments are based on analyses of Stark-difference spectra combined with the ground-state microwave data and the intensity variations which are expected theoretically. The ground and excited state $A, K=1$ asymmetry splitting parameters are measured to be $\delta_{1}^{\prime \prime}=(8.5450 \pm 0.0080) \times 10^{-3} \mathrm{~cm}^{-1}$ and $\delta_{1}^{\prime}$ $=(9.7706 \pm 0.0080) \times 10^{-3} \mathrm{~cm}^{-1}$, respectively. The ground-state value agrees well with the microwave results. A rapid-scan system for recording data and a computer-aided technique for calibrating and plotting the spectra are described.
\end{abstract}

\section{INTRODUCTION}

There has been considerable recent interest in high-resolution spectra of methyl alcohol, for both its normal and various isotopic forms $(l-4)$. The $\mathrm{C}-\mathrm{O}$ stretch fundamentals of these molecules overlap the $\mathrm{CO}_{2}$ laser emission band, thus they can be optically pumped with the $\mathrm{CO}_{2}$ laser, leading to far-infrared emission and laser action $(5-8)$. In this paper we report a tunable diode laser study of the $\mathrm{C}-\mathrm{O}$ stretch band of ${ }^{12} \mathrm{CD}_{3}{ }^{16} \mathrm{OH}$. This molcule is a slightly asymmetric rotor with a three-fold symmetric hindered internal rotation degree of freedom. The individual $P(J)$ and $R(J)$ multiplets of the $\mathrm{C}-\mathrm{O}$ stretch band are composed of $3 J$ and $3(J+1)$ components, respectively, each component of which is associated with the hindered rotation and $K$ substructure. The previous study of this band by Woods (9) using a grating spectrometer with $0.07-\mathrm{cm}^{-1}$ resolution led to assignments of the $J$ values of the multiplets, but none of the multiplet substructure was re- 
solved. We are able to resolve most of this structure and assign the transitions in the low- $J$ multiplets. The assignments are based primarily on the results of Starkdifference spectra, and they are generally compatible with the microwave groundstate measurements and the relative intensities expected theoretically.

Previous Stark effect measurements using tunable diode lasers have been reported on $\mathrm{NH}_{3}(l 0)$ and $\mathrm{CH}_{3} \mathrm{OH}(l)$. Sattler et al. (l) were able to assign several $\mathrm{CH}_{3} \mathrm{OH}$ lines from high-field Stark spectra in which all of the $\left|M_{J}\right|$ components were resolved. In $\mathrm{CD}_{3} \mathrm{OH}$, however, the intramultiplet spreading (typically 0.1 $\mathrm{cm}^{-1}$ ) is much reduced compared with $\mathrm{CH}_{3} \mathrm{OH}$ (typically $1.0 \mathrm{~cm}^{-1}$ ), and the individual $\left|M_{J}\right|$ components of the various transitions are badly overlapped with fields sufficiently high that they would be resolved for an isolated line. On the other hand, we have found that the systematics in the low-field Stark-difference spectra are sufficient to assign most of the lines in the low- $J$ multiplets, even when the lines are barely resolved with Doppler-limited resolution.

\section{EXPERIMENTAL DETAILS}

The experimental apparatus employed a Laser Analytics, Inc., diode laser in a double-beam system with two liquid $\mathrm{N}_{2}$ cooled $\mathrm{HgCdTe}$ detectors. The laser emission was first passed through a spectrometer and then split by a Ge-coated $\mathrm{BaF}_{2}$ beam splitter. One beam, used for calibration, passed through a $3.7879-\mathrm{cm}$ Ge etalon which produced fringes spaced by $0.03292 \mathrm{~cm}^{-1}$. The other beam passed through a $1-\mathrm{m}$ Stark cell containing the $\mathrm{CD}_{3} \mathrm{OH}$. Typical sample pressures were $0.1-0.3$ Torr and the plate spacing was $5 \mathrm{~mm}$. We used a repetitive, rapid-scan data acquisition method, which allowed the real-time observation of the spectra and etalon fringes on a dual-trace oscilloscope $(l /)$. The precise length and position of a particular scan could thus be set before recording. The data were then recorded on a 2048-channel signal averager at $10 \mu \mathrm{sec}$ per channel and transferred immediately to magnetic tape. Usually 64 or 128 sweeps were averaged and the records were $0.3-0.5 \mathrm{~cm}^{-1}$ in length.

In order to achieve Doppler-limited resolution for the averaged spectra, it was necessary to stabilize the laser frequency against slow drifts in the cold finger temperature. This was done with a feedback circuit that sampled the voltage from the calibration beam detector at some fixed delay after the start of the sweep, chosen to coincide with the steep part of one of the fringe peaks. This voltage was then compared with an adjustable reference voltage, and the difference was used to generate an error signal that was fed back to adjust the dc level of the diode laser current.

The relative frequency calibration was generated by a computer program that processed the fringe signal in the following manner. First a fringe pattern with normalized peak intensities was produced. The top $1 / 4$ of each fringe was leastsquares fitted to a parabola, the maximum of which was defined to be the location of the peak. Each maximum was assigned a frequency relative to some central fringe, and a cubic-spline interpolation routine (12) was used to establish a continuous frequency scale between maxima. Finally, a line-finding routine analyzed the sample spectrum and gave a printout of relative frequencies and intensities. Also, 
a frequency was assigned to each point in the spectrum, and these points were used as the abscissas in computer-generated plots such as those shown in the figures. Relative frequencies of isolated lines could be reproduced to within $0.0002-0.0005 \mathrm{~cm}^{-1}$, depending upon the signal-to-noise ratio. The computergenerated plots aided greatly in making the assignments since, as will be discussed later, there is a systematic pattern to the line positions that becomes apparent when successive $J$ multiplets are plotted on the same frequency scale and then overlaid.

\section{STARK EFFECT AND ASSIGNMENTS}

The rovibrational states of methanol-type molecules can be described in an $|n \tau K J\rangle$ representation in which $n$ is the torsional quantum number; $\tau=1,2,3$ designates the internal rotation state; and $K$ and $J$ are the usual angular momentum quantum numbers for a symmetric top $(13,14)$. An alternative to the $\tau$ notation, which is more convenient for discussing Stark effects, is the symmetry species designations of Lees and Baker (15): $A$ states correspond to $K+\tau=1, \bmod$ (3); $E_{1}$ states to $K+\tau=0, \bmod (3)$; and $E_{2}$ states to $K+\tau=2$, $\bmod (3)$. Asymmetry removes the $\pm K$ degeneracy of the $A$ states, which are split into symmetric and antisymmetric combinations of $\pm K$ ( $K$-doubling). The $E$ states remain doubly degenerate. The spin statistical weights arising from the identical deuteriums in the methyl group are in the ratio 11:8 for the $A$ to $E$ levels, which is thus the $A$ to $E$ intensity ratio of a given $J, K$ transition when the $K$-doubling is unresolved. When the $K$-doubling is resolved, as is always true for $K=1$, the $A$-to- $E$ ratio is 5.5:8(13).

For the purpose of interpreting the Stark-difference spectra, we need consider only the lowest-order Stark splittings. To lowest order, these splittings for $\mathrm{CD}_{3} \mathrm{OH}$ are approximated by those of a nearly symmetric rotor, i.e., the shift $\delta W$ of each $M_{J}$ component of a level is given by $(2,3,16,17)$

$$
\delta W=\frac{\mu_{a} \mathscr{E} K M_{J}}{J(J+1)}
$$

for the E states, and

$$
\delta W= \pm \frac{\Delta(J, K)}{2}\left[1-\left\{1+\frac{4 \mu_{a}^{2} \mathscr{E}^{2} K^{2} M_{J}^{2}}{\Delta(J, K)^{2} J^{2}(J+1)^{2}}\right\}^{1 / 2}\right]
$$

for the $A^{ \pm}$states, where $\mu_{a} \cong 0.9 \mathrm{D}$ is the component of the permanent dipole moment along the symmetry axis of the methyl group, $\mathscr{E}$ is the electric field, $M_{J}$ is the projection of $\mathbf{J}$ along $\mathscr{E}$, and $\Delta(J, K)$ is the asymmetry splitting of the $A$ levels. These asymmetry splittings for $K=1$ and $K=2$ are of the form

$$
\begin{aligned}
& \Delta(J, K=1)=\delta_{1} J(J+1), \\
& \Delta(J, K=2)=\delta_{2} J(J-1)(J+1)(J+2) .
\end{aligned}
$$

The values of $\delta_{1}$ and $\delta_{2}$ have been determined for the ground state from the microwave results of Lees and Baker (15): $\delta_{1}^{\prime \prime}=8.539 \times 10^{-3} \mathrm{~cm}^{-1}$ and $\delta_{2}^{\prime \prime}=1.92$ $\times 10^{-6} \mathrm{~cm}^{-1}$.

The selection rules for the $P$ and $R$ transitions in the $\mathrm{C}-\mathrm{O}$ stretch band are 


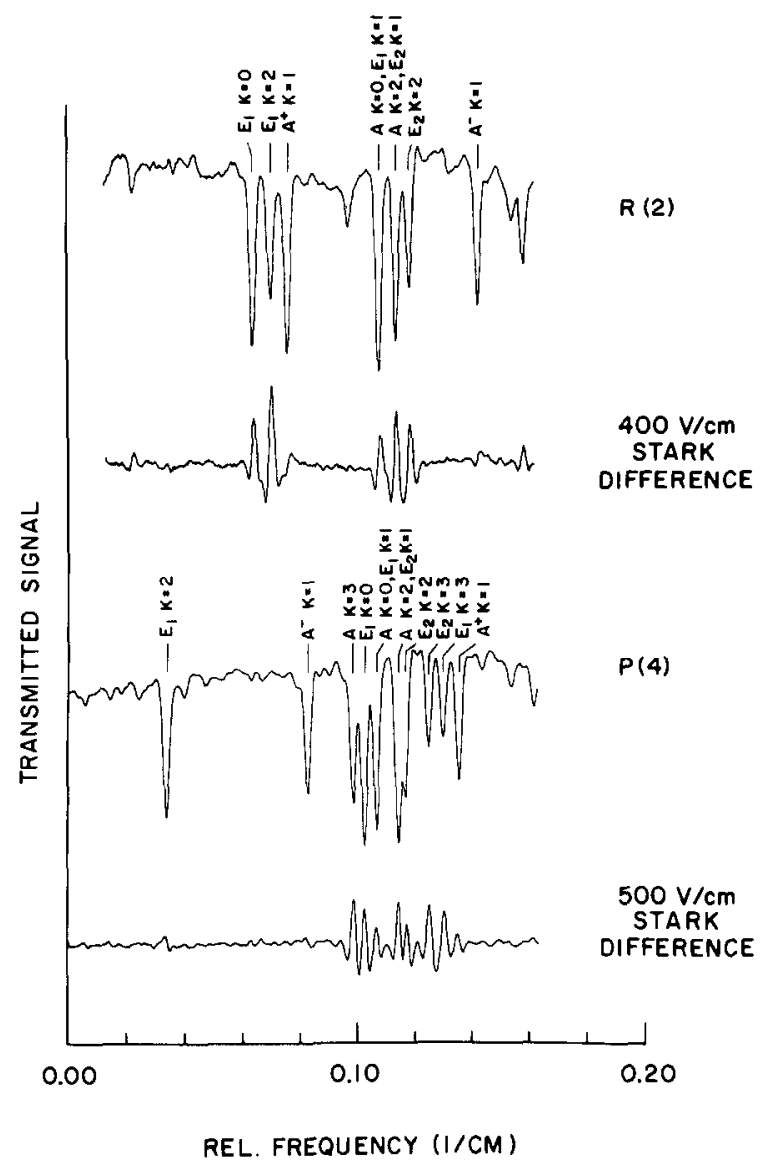

FIG. 1. Stark-difference spectra and zero-field spectra of $\mathrm{CD}_{3} \mathrm{OH}$ obtained with a tunable diode laser. The path length is $1 \mathrm{~m}$, the pressure is $0.3 \mathrm{Torr}$, and $\mathbf{E}_{\mathrm{Stark}}$ is $\perp$ to $\mathbf{E}_{\text {laser }}$. The absorption strength of the strongest lines is $10-20 \%$. The difference spectrum is obtained in each case by subtracting the zero-field spectrum from the field-on spectrum. The horizontal and vertical scales are preserved. The frequency of the $A, K=0$ line in $P(4)$ is $980.276 \mathrm{~cm}^{-1}$ and in $R(2)$ it is 989.397 , as based on a Fourier transform measurement of some nearby isolated lines.

$\Delta n=0, \Delta J= \pm 1, \Delta K=0, A \rightarrow A, E_{1} \rightarrow E_{1}, E_{2} \rightarrow E_{2}$. Since $K$ and the symmetry species do not change, we can use these parameters to distinguish the qualitative features of the Stark-difference spectra.

For a typical Stark field of $400-500 \mathrm{~V} / \mathrm{cm}$, the quantity $\mu_{a} \mathscr{E}$ is $\cong 6 \times 10^{-3} \mathrm{~cm}^{-1}$, which is substantially greater than the Doppler width $\cong 2.0 \times 10^{-3} \mathrm{~cm}^{-1}$. Thus the Stark-difference signals for the $E$ levels with $K=J$ will be a sizable fraction of the zero-field absorption strength, while the signals for levels with $K<J$ will be systematically reduced. Since $\mu_{a} \mathscr{E} \ll \Delta(J, 1)$, the $A, K=1$ levels will show a very small quadratic Stark splitting, and, in comparison to the $E$ levels of the same or larger $K$, their Stark-difference signals will be negligibly small. The $A, K=2$ levels for $J \lessgtr 4$ will show a linear Stark effect similar to that of the $E$ levels, 
but for larger $J$, they will be sufficiently split to show a quadratic Stark effect and thus a much reduced Stark-difference signal.

The $K=0$ levels show no first-order Stark effect. However, due to an accidental degeneracy the $E_{1}, K=0$ level (which is degenerate with $E_{2}, K=0$ ) is strongly admixed by asymmetry with the $E_{1}, K=2$ level (which is degenerate with $E_{2}, K=-2$ ). The admixed levels are strongly perturbed in position, in intensity, and in their Stark effects.

Examples of the data are shown in Fig. 1 for the $R(2)$ and $P(4)$ multiplets. The laser beam is linearly polarized in the direction perpendicular to the Stark field. The difference signals are obtained by digital subtraction of the zero-field transmission data from the field-on data. The scales for the difference signals are exactly the same as for the zero-field traces.

On the basis of the above discussion, the $A, K=1$ lines in Fig. 1 can be immediately assigned. In fact, the $A, K=1$ lines are readily assigned in all the multiplets, since they always appear as a pair of equal strength lines, symmetrically located with respect to the center of the multiplet, and they show no Stark-difference signal. The $A, K=0$ line is also readily assigned in all the multiplets, since it is the only line that shows no Stark splittings under high-field $\left(\sim 2 \times 10^{3} \mathrm{~V} / \mathrm{cm}\right)$ conditions.

The microwave data (15) provide combination difference relations between the $R(2)$ and $P(4)$ lines, since they have common upper states. Having established the assignments of the $A, K=1$ lines, we can use them to provide a relative frequency scale between the two multiplets, even though we have no adequate absolute calibration for either. This immediately allows us to assign several additional lines in these multiplets. The relative positions of the two spectra in Fig. 1 are adjusted so that the $A, K=0$ lines of each are at the same frequency. With this choice, the remaining lines in $R(2)$ (excepting $A, K=1$ and $E_{1}, K=0$, 2) occur at nearly the same position as the corresponding lines in $P(4)$. This is expected since the combination differences are all within $\sim 0.002 \mathrm{~cm}^{-1}$ of each other. The three lines in $P(4)$ which show the largest relative Stark-difference signals must belong to $K=3$. One of these is stronger than the other two by approximately the ratio $11: 8$; it must, therefore, be the $A, K=3$ transition. The distinction between $E_{1}$ and $E_{2}$ of the remaining two lines is based on a slightly better fit to the combination differences used in the analysis of the $R(3)$ and $P(5)$ transitions.

The $A, K=2$ and $E_{2,}, K=2$ lines in $R(2)$ are identified by their large Starkdifference signals. On the basis of the relative intensities and by the process of elimination, we conclude that the $E, K=1$ lines must overlap the $A, K=0$ and $A, K=2$ lines. The choice shown in the figure of putting the $E_{1}$ line with $A$, $K=0$ and $E_{2}$ with $A, K=2$ is based on a slightly better fit to the combination relations. This choice must remain tentative at present, since the intensities and Stark effect do not distinguish $E_{1}$ from $E_{2}$, and these lines are always blended so the combination differences are not definitive.

Figures 2 and 3 summarize the data obtained for the first five $P$ - and $R$-branch transitions. The spectra are all plotted with the position of $A, K=0$ at $0.12 \mathrm{~cm}^{-1}$. The $P(2)$ transition falls in the dense $Q$-branch region and we were unable to find 


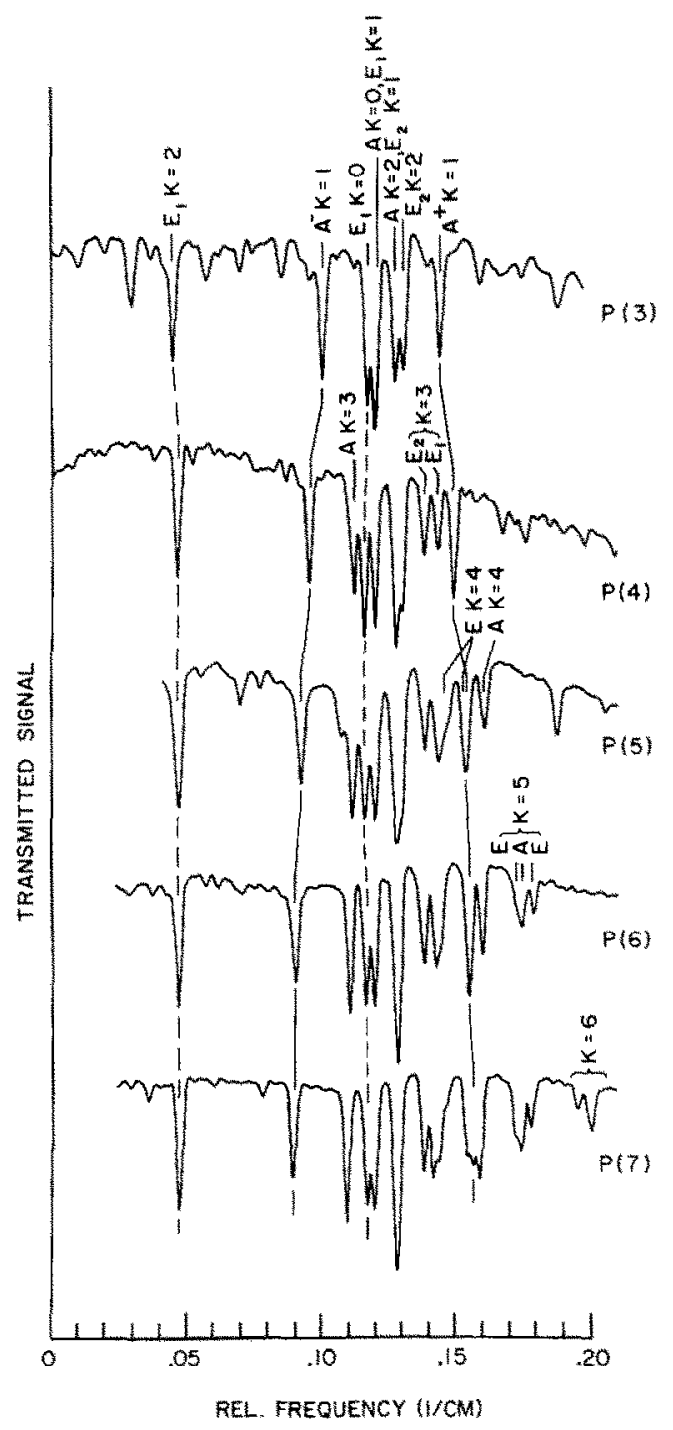

Fig. 2. $P$-branch lines of $\mathrm{CD}_{3} \mathrm{OH}$ recorded with a diode laser and then plotted with the aid of a computer on a calibrated relative frequency axis. The relative intensity between multiplets is not preserved. The $A, K=0$ lines oceurs at $0.12 \mathrm{~cm}^{-1}$ in each plot.

it. All of the spectra, excepting $P(5)$, show Doppler-broadened linewidths. The widths in $P(5)$ are about $50 \%$ broader due to a lower signal-to-noise ratio, which degraded the laser frequency stabilization. The assignments indicated in these figures were made using the above arguments. The progression of the $A, K=1$ lines is indicated by solid lines and that for the perturbed $E_{1}, K=0$ and $K=2$ lines by dashed lines. The $K$ designations for these latter transitions are the same as those used by Lees and Baker (15), even though $K$ is not a good quantum number for these strongly admixed states. To simplify the figures, the remaining lines 
are indicated only when they first appear in a multiplet. In higher- $J$ multiplets, the same lines appear at nearly the same relative frequency. This feature was pointed out by Sattler et al. (1) in their analysis of $\mathrm{CH}_{3} \mathrm{OH}$ and it is also quite apparent in the $\mathrm{CD}_{3} \mathrm{OH}$ spectra.

The $A, K=1$ asymmetry splitting parameters $\delta_{1}^{\prime \prime}$ and $\delta_{1}^{\prime}$ for the ground and excited states can be determined directly from our data using combination differences. For the ground state we use

$R\left(A^{-}, J-1\right)-R\left(A^{+}, J-1\right)-P\left(A^{-}, J+1\right)+P\left(A^{+}, J+1\right)=(4 J+2) \delta_{1}^{\prime \prime}$,

and for the excited state

$$
R\left(A^{-}, J\right)-R\left(A^{+}, J\right)-P\left(A^{-}, J\right)+P\left(A^{+}, J\right)=(4 J+2) \delta_{1}^{\prime}
$$

A least-squares fit of our data to these equations yields

$$
\begin{aligned}
& \delta_{1}^{\prime \prime}=(8.5450 \pm 0.0080) \times 10^{-3} \mathrm{~cm}^{-1}, \\
& \delta_{1}^{\prime}=(9.7706 \pm 0.0080) \times 10^{-3} \mathrm{~cm}^{-1} .
\end{aligned}
$$

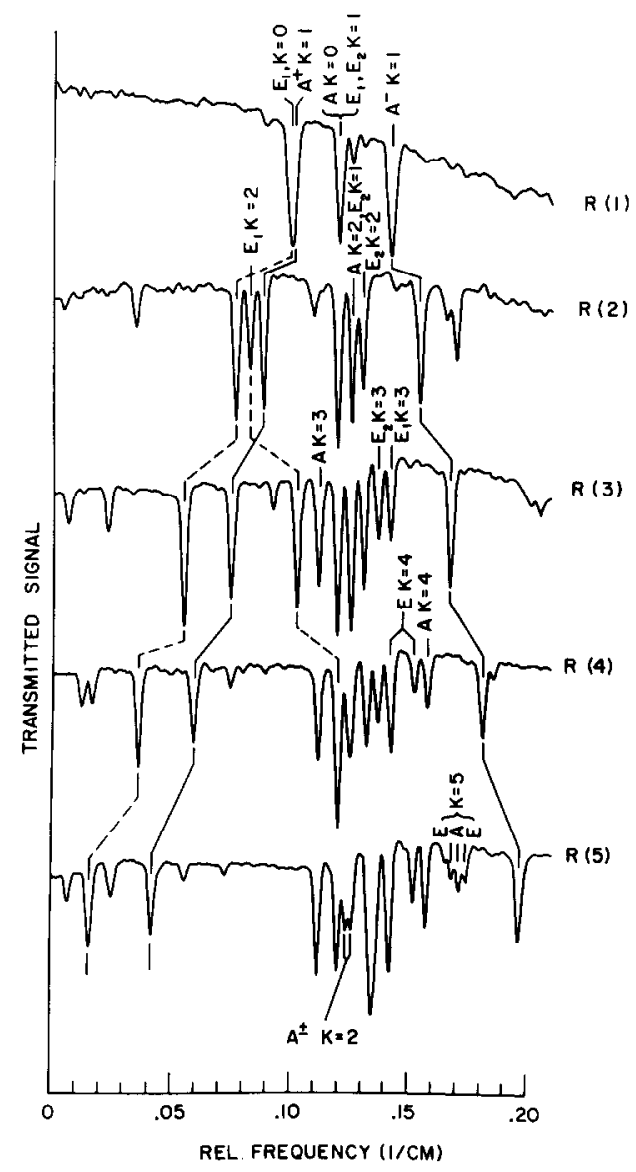

FIG. 3. $R$-branch lines of $\mathrm{CD}_{3} \mathrm{OH}$ obtained in the same way as the spectra in Fig. 2. 
The uncertainties are $2 \sigma$ estimates. The rms fit to Eq. (4) was $\pm 0.00036 \mathrm{~cm}^{-1}$ for six points and to Eq. (5), $\pm 0.00059 \mathrm{~cm}^{-1}$ for seven points. Our value for $\delta_{1}^{\prime \prime}$ agrees well with the microwave value, $8.539 \times 10^{-3} \mathrm{~cm}^{-1}$.

We have not been able to determine the $A, K=2$ asymmetry splitting. This splitting is not resolved in any of the $P$ lines. In the $R$ lines it first appears as a broadening in $R(4)$ and then as a clearly resolved doublet in $R(5)$. In the higher $R(J)$ lines, through $R(10)$, one or the other of the $A^{ \pm}, K=2$ components is overlapped. On the basis of the splitting observed in $R(5)$ we can conclude that this asymmetry splitting in the excited state is within about $10 \%$ of the groundstate value.

The barrier height $H$ of the hindered rotation potential changes slightly in the vibrational excited state. If we ignore the Kirtman interaction terms, this barrier height change can be roughly estimated from the frequency difference between individual lines in a $J$ multiplet which are not affected by asymmetry. On this basis, we estimated that $\Delta H$ is between -1 and $-2 \mathrm{~cm}^{-1}$, which corresponds to a decrease in $H$ of $\approx 0.5 \%$.

Although most of the assignments presented here are undoubtedly correct, there is a great deal of overlapping and some of the identifications must remain tentative. For example, all of the lines in $R(1)$ appear to be blended and the $A^{-}$, $K=1$ line appears too strong. This line may be overlapped with the "forbidden" transition $E_{1}, K=2 \leftarrow E_{1}, K=0$, which becomes allowed because of the admixture of $K=0$ into the upper state. Overlapping is also a severe problem near the centers of most of the high- $J$ multiplets. We are presently attempting to establish an absolute frequency calibration for the data and with that to obtain a fit to the spectrum based on the Hamiltonian used by Kwan and Dennison (/3).

\section{ACKNOWLEDGMENTS}

The authors are indebted to B. D. Poindexter for assistance in recording the data, to C. F. Eagen and L. A. Feldkamp for assistance in obtaining the computer generated spectral plots, to D. G. Snyder for providing us with a set of computed ground-state energies to augment the microwave data, and to P. D. Maker for recording a Fourier transform spectrum.

Note added in proof. A preliminary fit of our spectra to the Hamiltonian used by Kwan and Dennison indicates that the barrier height change is even less than estimated above and that the tentative assignments shown in the figures for the $E_{1}$ and $E_{2}, K=1$ transitions should be interchanged.

RECEIVED: February 16, 1981

\section{REFERENCES}

1. J. P. Sattler, W. A. Riessler, And T. L. Worchesky, Infrared Phys. 19, 217-224 (1979): 18, 521-528 (1978).

2. J. O. Henningsen, J. Mol. Spectrosc. 83, 70-93 (1980).

3. L. H. Johnston, R. P. Srivastaya, and R. M. Lees, J. Mol. Spectrosc. 84, 1-40 (1980).

4. J. O. Henningisen, J. C. Petersen, F. R. Petersen, D. A. Jennings. and K. M. Evenson, J. Mol. Spectrosc. 77, 298-309 (1979).

5. T. Y. Chang and T. J. Bridges, Opt. Commun. 1, 423-426 (1970).

6. E. J. Danielewicz and P. D. Coleman, IEEE J. Quantum Electron. QE-13, 485-490 (1977). 
7. E. J. Danielewicz and C. O. Weiss, IEEE J. Quantum Electron. QE-14, 458-459 (1978).

8. S. F. Dyubko, V. A. Svich, AND L. D. Fesenko, Radiofizika 18, 1434-1437 (1975).

9. D. R. Woods, Ph.D. Thesis, University of Michigan, Ann Arbor, 1970.

10. W. H. Weber, P. D. Maker, K. F. Yeung, and C. W. Peters, Appl. Opt. 13, 1431-1434 (1974).

11. W. H. Weber, D. H. Leslie, C. W. Peters, and R. W. Terhune, J. Mol. Spectrosc. 81, $316-326(1980)$.

12. "The International Mathematical and Statistical Library," Vol. 2, Chap. I, IMSL, Inc., Houston, 1980.

13. Y. Y. KWAN AND D. M. Dennison, J. Mol. Spectrosc. 43, 291-319 (1972).

14. D. G. Burkhard and D. M. Dennison, Phys. Rev. 84, 408-417 (1951).

15. R. M. Lees and J. G. Baker, J. Chem. Phys. 48, 5299-5318 (1968).

16. C. H. Townes and A. L. Schawlow, "Microwave Spectroscopy," Chap. 10, McGraw-Hill, New York, 1955.

17. E. V. Ivash And D. M. Dennison, J. Chem. Phys. 21, 1804-1816 (1953). 